\title{
A Multi-Parameter Decoupling Method with a Lamb Wave Sensor for Improving the Selectivity of Label-Free Liquid Detection ${ }^{\dagger}$
}

Lianqun Zhou ${ }^{1,2,3}$, Yihui Wu ${ }^{1,2, *}$, Ming Xuan ${ }^{1,2}$, Jean-François Manceau ${ }^{3}$ and François Bastien ${ }^{3}$

1 Suzhou Institute of Biomedical Engineering and Technology, Chinese Academy of Sciences, Suzhou 215163, China; E-Mails: zhoulq@sibet.ac.cn (L.Z.); xuanm@ciomp.ac.cn (M.X.)

2 State Key Laboratory of Applied Optics, Changchun Institute of Optics, Fine Mechanics and Physics, Chinese Academy of Sciences, Changchun 130033, China

3 Institute FEMTO-ST, CNRS, Université de Franche-Comté, Besançon 25044, France; E-Mails: jfmanceau@femto-st.fr (J.-F.M.); fbastien@femto-st.fr (F.B.)

$\dagger$ Earlier version of this paper appears in the 16th International Conference on Solid-State Sensors, Actuators and Microsystems, Beijing, China, 5-9 June 2011.

* Author to whom correspondence should be addressed; E-Mail: yihuiwu@ciomp.ac.cn; Tel.: +86-512-6958-8061; Fax: +86-512-6958-8058.

Received: 17 June 2012; in revised form: 17 July 2012 / Accepted: 24 July 2012 /

Published: 31 July 2012

Abstract: In this paper, a liquid multi-parameter decoupling method with only one Lamb wave sensor is presented. In a Lamb wave sensor, antisymmetric modes $\left(\mathrm{A}_{01}\right.$ mode for low frequency, $\mathrm{A}_{03}$ mode for high frequency) and symmetric modes ( $\mathrm{S}_{0}$ mode) are used to detect multiple parameters of a liquid, such as its density, sound velocity, and viscosity. We found they can play very different roles in the detections. For example, the $\mathrm{A}_{01}$ mode is very sensitive to the liquid's density but the $\mathrm{A}_{03}$ mode is sensitive to the sound velocity. Here, the $A_{0}$ mode is used to identify the density of the detected liquid and with this density value we obtained the viscosity by the amplitude shifts of the $\mathrm{S}_{0}$ mode. This could be a way to distinguish an unknown liquid with high sensitivity or to solve the problem of selectivity of label-free detection on biosensors.

Keywords: Lamb wave sensor; density; sound velocity; viscosity; determination of multi-parameters 


\section{Introduction}

Acoustic sensors have been widely used in chemical/biological fields with the label-free detection method to detect the mass changes on the sensor surface [1-17]. However, the selectivity of this method is often poor due to the absorption of non-target molecules, which are difficult to distinguish using only one-parameter sensors [18,19]. This has been a common problem of all kinds of label-free biosensors. The detection of multiple parameters using a multi-mode acoustic sensor significantly improves the label-free detection method. Acoustic waves which travel in a medium can have multiple modes and this character has been successfully used in a combined detection of density and viscosity for high viscosity solutions [20]. However, for low viscosity solutions like aqueous electrolytic solutions or bio liquids, it is still a challenge [9,20-24].

The micro Lamb wave sensor is a powerful tool for liquid detection because it is easier to get multi-mode vibration, and it performs with high sensitivity and low attenuation [25-29]. The two well-known basic modes, the antisymmetric mode $\left(\mathrm{A}_{0}\right.$ mode) and the symmetric mode $\left(\mathrm{S}_{0}\right.$ mode $)$, have already been used for solving the problem of temperature compensation on a chip by the authors of $[26,30]$. The $\mathrm{A}_{0}$ mode has been used to measure the concentration of bio/chemical liquids, such as the concentration of methanol for direct methanol cell applications [18]. However, it shows uncertain frequency shifts direction with the concentration changes of the liquid [18,31]. In fact, the parameters of a liquid, like the density, acoustic sound velocity and viscosity, will work together for a mode. So it's hard to decouple them with only one mode.

In this work, both the antisymmetric mode and the symmetric modes are used to decouple the liquid physical parameters. The characters of $\mathrm{A}_{03}$ mode, with a wavelength of about one-third of $\mathrm{A}_{01}$ mode (low frequency of $\mathrm{A}_{0}$ mode), are investigated with the liquid loading for the first time. In order to discuss the response of Lamb wave sensor to the liquid loading, two types of experiments were set up. The first experiment was used to measure the frequency shifts with the same type of solutions with different concentrations loaded on the sensors' surface, such as the solutions of $\mathrm{NaCl}$. It shows that the characters of the modes are very different. Although both $\mathrm{A}_{01}$ and $\mathrm{A}_{03}$ modes belong to $\mathrm{A}_{0}$ mode, their frequency shifts showed an opposite behavior with the increase of the concentration of the loading solutions. The second experiment was used to measure the frequency changes with different types of solutions and different concentrations, such as solutions of $\mathrm{KCl}, \mathrm{NaBr}$ and $\mathrm{KBr}$. Although the tested objects are two different kinds of solutions with different concentrations, the frequency of $\mathrm{A}_{01}$ mode shows almost has no movement and $\mathrm{A}_{03}$ mode frequency shows a great difference. The frequency shifts of $\mathrm{S}_{0}$ mode for these solutions are not apparent. These will make it possible to be only sensitive to the liquid viscosity except for the environmental temperature. This essay attempts to decouple the functions of $\mathrm{A}_{01}, \mathrm{~A}_{03}$ and $\mathrm{S}_{0}$ modes to obtain the basic physical parameters (density and sound velocity, viscosity) with different liquid loading responses. From the values which have been reported from literature [32] we can also determine the types of the solution. This work provides a selective, sensitive method for the measurement of physical parameters to an unknown solution. It can be useful for label-free biosensors. 


\section{Working Principle}

Multi-mode wave, such as $\mathrm{A}_{01}$ mode, $\mathrm{S}_{0}$ mode and their harmonic ones ( $\mathrm{A}_{03}$ mode, for example) can be excited in Lamb wave sensors and detected directly by using a pair of inter-digital transducers (IDTs) [33] located on the surface of the piezoelectric layer [34,35]. In liquid sensing, the liquid and IDT will be located on the two opposite sides of the membrane. For the $A_{01}$ mode and the $A_{03}$ mode, when the Lamb wave phase velocity is less than the liquid sound velocity, there are evanescent waves produced around the membrane-liquid interface. The phase velocity of the Lamb waves within the evanescent penetration field will be the same with the phase velocity inside the membrane. Taking account of the bending stiffness and the in-plane tension of the plate $\left(\mathrm{B}_{\mathrm{i}}\right)$ for the $\mathrm{A}_{01}$ mode and the $\mathrm{A}_{03}$ mode, the phase velocity will be [31,36]:

$$
c_{P i}=\sqrt{B_{i} /\left(M+m_{L i}\right)}
$$

where $i$ denotes 1 or 3 for the $\mathrm{A}_{0 \mathrm{i}}$ mode, $B_{\mathrm{i}}$ reflects the influences of the bending stiffness and the in-plane tension of the plate for the $\mathrm{A}_{0 \mathrm{i}}$ mode, $M$ is the mass per unit area of the plate, the effective mass $m_{L i}$ in the so-called evanescent penetration depth $\delta_{E i}$ equals:

$$
m_{L i}=\rho_{L} \delta_{E i}
$$

In which, $\rho_{L}$ is the detected liquid density. The evanescent penetration depth $\delta_{E i}$ obeys the following equation [37-39]:

$$
\delta_{E i}=\lambda_{i} /\left(2 \pi \sqrt{1-\left(c_{P i} / c_{L}\right)^{2}}\right)
$$

where $\lambda_{i}$ and $c_{P i}$ denote the Lamb wave wavelength and phase velocity respectively in each mode. $c_{L}$ is the bulk acoustic velocity of the detected liquid. $c_{P i}$ is determined by the frequency $\left(f_{i}\right)$ via $c_{P i}=f_{i} \lambda_{i}$.

By substituting the Equations (2) and (3) into the Equation (1), we will get the following formula:

$$
\rho_{L} \lambda_{i} /\left(2 \pi \sqrt{1-\left(c_{P i} / c_{L}\right)^{2}}\right)=B_{i} / c_{P i}^{2}-M
$$

In this formula, the constants $B_{i}, \lambda_{i}$, and $M$ are independent of the liquid type; $\lambda_{i}$ is determined by the structure of the device. With the implicit Equation (4), the density and sound velocity of the liquid on the sensor can be decoupled when the frequency response of the $\mathrm{A}_{01}$ mode and the $\mathrm{A}_{03}$ mode are obtained by the experiments.

In the measurement, the relative frequency shifts $(\Delta f / f)$ will be used frequently. By taking the term $c_{P i}=f_{i} \lambda_{i}$ into Equation (1) and deriving of the equation, the value of $\Delta f f f$ ) for the $\mathrm{A}_{01}$ mode and the $\mathrm{A}_{03}$ mode will be:

$$
\Delta f_{i} / f_{i}=-\Delta\left(\rho_{L} \delta_{E i}\right) / 2 M=-\left(\delta_{E i} \Delta \rho_{L}+\rho_{L} \Delta \delta_{E i}\right) / 2 M
$$

Obviously, the density and the sound velocity are the two factors affecting the relative frequency shifts. When the phase velocity is far less than the liquid sound velocity ( $\left.c_{P} \ll c_{L}\right)$, such as the $\mathrm{A}_{01}$ mode, the values of $\delta_{E}$ and $\Delta \delta_{E}$ approach $\lambda / 2 \pi$ and 0 , respectively. Then, in this situation the variations of the liquid sound velocity will have an almost negligible influence on the relative frequency shift. When the phase velocity is close to the liquid sound velocity, such as the $\mathrm{A}_{03}$ mode, the value $1-\left(c_{P} / c_{L}\right)^{2}$ approaches 0 . The influence of the terms $\delta_{E}$ and $c_{L}$ cannot be neglected any more. The 
liquid density and sound velocity will simultaneously affect the frequency shifts. The value of $\Delta f / f$ can be positive or negative which depends on the sum of the value of $\rho_{L} \Delta \delta_{E}$ and the value of $\delta_{E} \Delta \rho_{L}$. Anyway, if we combine these two cases $\left(\mathrm{A}_{01}\right.$ mode, $\mathrm{A}_{03}$ mode) simultaneously, we can get the values $\rho_{L}$ and $c_{L}$ with high sensitivity.

For the $\mathrm{S}_{0}$ mode, when the wavelength of the Lamb mode is larger than the thickness of the plate, the phase velocity $\left(c_{p}\right)$ for the principal symmetric mode can be simplified as [40]:

$$
c_{p}=c_{t}\left[4\left(1-\frac{c_{t}^{2}}{c_{d}^{2}}\right)\right]^{\frac{1}{2}}\left[1+\frac{I}{2} \frac{\rho_{L} c_{L}^{2}}{\rho_{m} c_{t}^{2}} \frac{\omega d}{2 c_{L}}\left(\frac{1}{4\left(1-c_{t}^{2} / c_{d}^{2}\right)}-\frac{c_{t}^{2}}{c_{d}^{2}}\right)\right]
$$

where $c_{d}$ and $c_{t}$ are the velocities of longitudinal (dilatational) and transverse (shear) waves of the membrane, $c_{L}$ is the liquid sound velocity, $\rho_{m}$ and $\rho_{L}$ are the density of the solid membrane and liquid respectively, $d$ is the membrane thickness, $I$ denotes the square root of -1 .

Evidently, the effect of liquid on the propagation of the $\mathrm{S}_{0}$ mode does not change the real part of the phase velocity, but adds a very small attenuation of the amplitude [40]. This mode is suitable for the detection of attenuation. The amplitude $\left(A_{L}\right)$ response of an unknown solution can be expressed by:

$$
A_{L}=A_{0} e^{-\alpha_{X L} x}
$$

where the attenuation coefficient $\alpha_{X L}$ is proportional to $\left(\rho_{L} \eta_{L}\right)^{0.5}$, with $\eta_{L}$ being the viscosity of the liquid. Similarly, the amplitude response $\left(A_{W}\right)$ of water is given by:

$$
A_{W}=A_{0} e^{-\alpha_{X W} x}
$$

In engineering, the insertion loss (dB) is widely used. The values of $A_{L}$ and $A_{W}$ can be transformed into values in $\mathrm{dB}$ scale which are denoted by $A_{L d B}$ and $A_{W d B}$, respectively. Therefore, the attenuation difference $\left(\triangle A_{d B}\right)$ between an unknown solution and water can be expressed in $\mathrm{dB}$ scale as follows:

$$
\Delta A_{d B}=A_{L d B}-A_{W d B}=\gamma_{1}\left(\rho_{L} \eta_{L}\right)^{1 / 2}+\gamma_{2}
$$

in which both of the slope $\gamma_{1}$ and $\gamma_{2}$ are constant, and the constant $\gamma_{2}$ is determined by the reference liquid (water). As the density and the sound velocity of the solution can be estimated by both the frequency shifts of the $\mathrm{A}_{01}$ mode and the $\mathrm{A}_{03}$ mode, the multi-parameters $\left(\rho_{L}, c_{L}, \eta_{L}\right)$ of the solution can be decoupled simultaneously with these three modes. According to these principles, a series of experiments were set up to investigate the responses of the multi modes of Lamb wave to the loading solutions.

\section{Experiments for Liquid Detection}

\subsection{Experimental Setup}

The micro Lamb wave device in Figure 1(a) contains a silicon membrane (length $7.8 \mathrm{~mm}$, thickness $\sim 12 \mu \mathrm{m}$ ) with a ground layer (Ti/Mo, GND, $\sim 0.2 \mu \mathrm{m}$ ) and a piezoelectric layer (aluminum nitride, AlN, $\sim 1.8 \mu \mathrm{m})$. Lamb waves are excited and detected directly using bidirectional inter-digital transducers (IDTs) [33] located on the surface of the AlN layer. There are six pairs of fingers on bidirectional IDTs in each exciting and detecting transducer. The period of bidirectional IDT is about $400 \mu \mathrm{m}$. As waves are partly reflected at the end of length-limited membrane $(\sim 7.8 \mathrm{~mm})$, the device 
has strong signal without reflectors on both ends of the membrane. With the layers described as in [34], the mass per unit area of the membrane $(M)$ is about $0.0355 \mathrm{~kg} / \mathrm{m}^{2}$.

The micro Lamb wave device is packaged directly with the printed circuit board (PCB), as shown in Figure 1(b). The network analyzer (Agilent 4395A), connected with the PCB, is used to excite and receive the acoustic signals. The device is protected with one cover on top of the system. The tube is used to pass the liquid into/out of the chamber, which is sealed up with the polymethyl methacrylate (PMMA) cover under the PCB.

Figure 1. The system for liquid detection, (a) Schematic diagram of the micro Lamb wave sensor interaction with liquid, $\rho_{L}$ : density, $c_{L}$ : sound velocity, $\eta_{L}$ : viscosity; (b) Micro Lamb wave sensor packaged with printed circuit board (PCB).

(a)

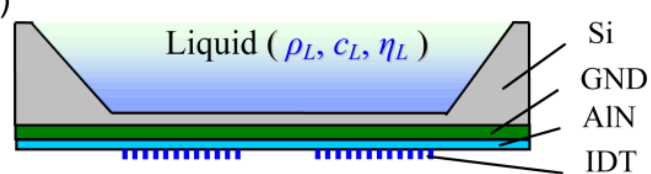

(b)

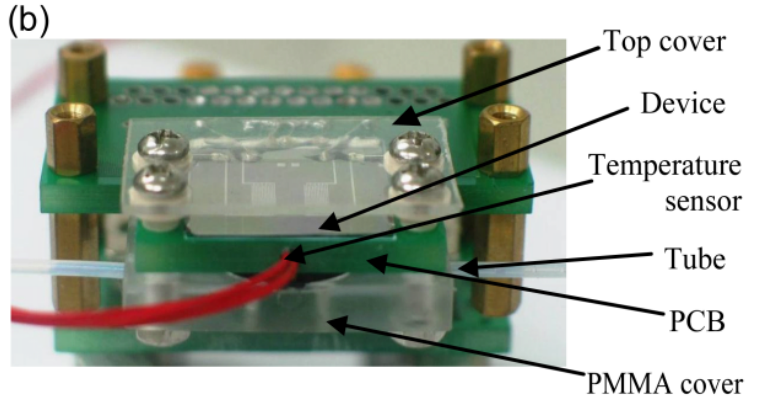

Figure 2. The responses of the $A_{01}$ mode, the $A_{03}$ mode, and the $S_{0}$ mode to the air and the water loading on the Lamb wave sensor.

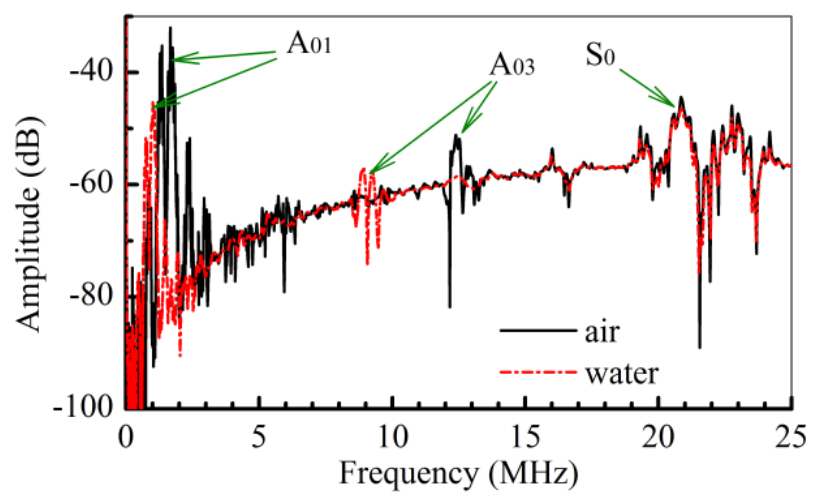

When the device is loaded with air or water, multi-modes can be excited and detected effectively, including the $\mathrm{A}_{01}$ mode, the $\mathrm{A}_{03}$ mode, and the $\mathrm{S}_{0}$ mode (Figure 2). As the $\mathrm{A}_{01}$ and $\mathrm{A}_{03}$ modes are harmonic, the wavelength of the $\mathrm{A}_{03}$ mode is about one third of the wavelength of the $\mathrm{A}_{01}$ mode. According to the positions of the $\mathrm{A}_{01}$ mode, $\mathrm{A}_{03}$ mode and $\mathrm{S}_{0}$ mode in the dispersion curves [41,42], the resonant frequencies $(f)$ of these three modes should have the following relation: $f_{A 01}<f_{A 03}<f_{S 0}$. The measured resonant frequency of the $\mathrm{A}_{01}$ and $\mathrm{A}_{03}$ modes for water loading are 0.987 and $9.233 \mathrm{MHz}$, respectively. With the frequency $(f)$ and the wavelength $(\lambda)$, the corresponding phase velocities $(f \lambda)$ of 
these two modes are $385 \mathrm{~m} / \mathrm{s}$ and 1,200 m/s respectively. Although the phase velocity of the $\mathrm{A}_{01}$ mode is far less than the sound velocity of water $(1,485.5 \mathrm{~m} / \mathrm{s})$, it is close to the sound velocity of water for the $\mathrm{A}_{03}$ mode. With the same water loading for the $\mathrm{S}_{0}$ mode, the measured central resonant frequency is about $20.86 \mathrm{MHz}$. The corresponding phase velocity of the $\mathrm{S}_{0}$ mode is about $8,135.4 \mathrm{~m} / \mathrm{s}$. Further experiments were conducted to investigate the response of multi-modes to the different solutions.

\subsection{Experiments for One Species Solutions (NaCl Solutions) with Different Concentrations}

The first experiment was done to measure known solutions with different concentrations (Figure 3), such as $\mathrm{NaCl}$ solutions. When water is designated as the reference liquid, the relative frequency shifts $\left(\Delta f f=\left(f_{\text {solution }}-f_{\text {water }}\right) / f_{\text {water }}\right)$ with concentration are different for these three modes $\left(\mathrm{A}_{01}\right.$ mode, $\mathrm{A}_{03}$ mode, and $\mathrm{S}_{0}$ mode), where $f_{\text {solution }}$ and $f_{\text {water }}$ are the measured frequencies for the solution and the water, respectively. The frequency of the $\mathrm{A}_{01}$ mode decreases with the concentrations and the $\mathrm{A}_{03}$ mode behaves oppositely. In the case of the $\mathrm{A}_{03}$ mode of $\mathrm{NaCl}$ solutions measured in Figure 3, the absolute value $\rho \Delta \delta_{E}$ is bigger than the absolute value $\delta_{E} \Delta \rho$ (Table 1). This causes the positive frequency shifts of $\mathrm{A}_{03}$ mode in $\mathrm{NaCl}$ solutions measurements. In any case, when the frequency shift is positive, this means the Lamb wave phase velocity is close to the liquid sound velocity. In this case, this phenomenon can be used to decouple the liquid sound velocity.

Figure 3. Relative frequency shifts $(\Delta f / f)$ for the $\mathrm{A}_{01}$ mode, the $\mathrm{A}_{03}$ mode, and the $\mathrm{S}_{0}$ mode in the measurements of the $\mathrm{NaCl}$ solutions.

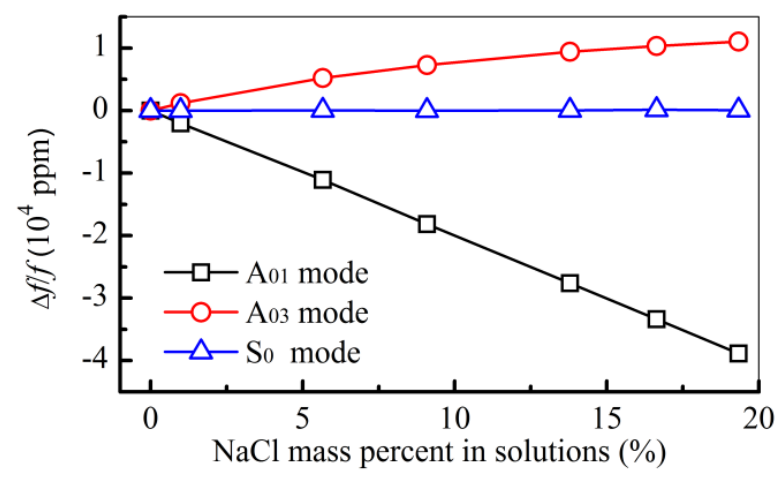

Table 1. Based on the measured frequency shifts of $\mathrm{NaCl}$ solutions measured in Figure 3, comparison of the value $\rho \Delta \delta_{E}$ and the value $\delta_{E} \Delta \rho$ for the $\mathrm{A}_{03}$ mode.

\begin{tabular}{cccccc}
\hline Concentration $\mathbf{( \% )}$ & Density $\left(\mathbf{1 0}^{\mathbf{3}} \mathbf{~ k g} / \mathbf{m}^{\mathbf{3}}\right)$ & $c_{P}(\mathbf{m} / \mathbf{s})$ & $\delta_{E}(\boldsymbol{\mu m})$ & $\delta_{E} \Delta \rho$ & $\rho \Delta \delta_{E}$ \\
\hline 0 & 0.9981 & $1,200.30$ & 35.12 & 0 & 0 \\
0.99 & 1.0052 & $1,201.71$ & 34.70 & 0.25 & -0.42 \\
5.67 & 1.0389 & $1,206.55$ & 32.81 & 1.34 & -2.39 \\
9.09 & 1.0640 & $1,209.03$ & 31.58 & 2.08 & -3.77 \\
13.79 & 1.0995 & $1,211.59$ & 30.01 & 3.04 & -5.62 \\
16.64 & 1.1212 & $1,212.69$ & 29.13 & 3.59 & -6.71 \\
19.33 & 1.1425 & $1,213.53$ & 28.40 & 4.10 & -7.67 \\
\hline
\end{tabular}

Compared with the frequency shifts measured with the $A_{01}$ mode and $A_{03}$ mode, the frequency of the $\mathrm{S}_{0}$ mode shows negligible shift with different concentrations (Figure 3 ). 


\subsection{Experiments for Three Different Unknown Species Solutions}

The second experiment was done to measure the modes' responses to three different species of aqueous electrolytic solutions with different concentrations (Table 2). We will check the possibility of the method to decouple the density and the acoustic sound velocity of these solutions with the measurements of $\mathrm{A}_{01}$ mode and $\mathrm{A}_{03}$ mode. Three different species of aqueous electrolytic solutions are prepared, they are listed as solution A, solution B, and solution $\mathrm{C}$ respectively, as it is shown in Table 2 . From No. 1 to No. 6 solutions, they are the solution A. No. 7 and No. 8 solutions are the solution B. No. 9 and No. 10 are solution C. In each kind of solution, the concentration increases with the serial number. The $\mathrm{S}_{0}$ mode is still insensitive to the solution changes compared with the measurement in water, and its values are not listed here.

Table 2. Relative frequency shifts $(\Delta \mathrm{f} / \mathrm{f})$ for the $\mathrm{A}_{01}$ mode and the $\mathrm{A}_{03}$ mode in the measurements of three kinds of unknown solutions. In each kind solution, the concentration increases with the serial number.

\begin{tabular}{|c|c|c|c|c|c|c|c|c|c|c|}
\hline \multirow[b]{2}{*}{ No. of solutions } & \multicolumn{6}{|c|}{ Solution A } & \multicolumn{2}{|c|}{ Solution B } & \multicolumn{2}{|c|}{ Solution C } \\
\hline & 1 & 2 & 3 & 4 & 5 & 6 & 7 & 8 & 9 & 10 \\
\hline Concentration (\%) & 0.99 & 6.05 & 9.97 & 16.03 & 19.96 & 23.95 & 9.35 & 18.07 & 8.98 & 17.32 \\
\hline $\begin{array}{l}\Delta f / f\left(\mathrm{~A}_{01} \text { mode }\right) \\
\left(-1 \times 10^{4} \mathrm{ppm}\right)\end{array}$ & 0.236 & 1.09 & 1.846 & 2.886 & 3.654 & 4.434 & 2.243 & 4.474 & 2.216 & 4.356 \\
\hline $\begin{array}{c}\Delta f / f\left(\mathrm{~A}_{03} \text { mode }\right) \\
\left(10^{4} \mathrm{ppm}\right)\end{array}$ & 0.027 & 0.216 & 0.357 & 0.373 & 0.325 & 0.287 & -0.649 & -1.422 & -0.876 & -1.848 \\
\hline
\end{tabular}

In the case of solution $\mathrm{A}$, the relative frequency shifts $\Delta f / f$ of $\mathrm{A}_{01}$ mode and $\mathrm{A}_{03}$ mode have similar properties with the solution of $\mathrm{NaCl}$ measured in Figure 3. But for the solution $\mathrm{B}$ and solution $\mathrm{C}$, the relative frequency shifts of $\mathrm{A}_{03}$ mode is negative. As it is indicated in Equation (5), the absolute value $\rho \Delta \delta_{E}$ of solution B and solution C measured in Table 2 should be smaller than the absolute value $\delta_{E} \Delta \rho$.

Even for different kinds of solutions, such as the No. 6, No. 8, and No. 10 solutions, the values of $\Delta f / f$ of the $\mathrm{A}_{01}$ mode are almost the same, but the values of $\Delta f f f$ of the $\mathrm{A}_{03}$ mode are apparently different. This means only one antisymmetric mode measurement, such as $\mathrm{A}_{01}$ mode, can not determine the solution with high selectivity. In order to specify the liquid with high selectivity, it is better to decouple the liquid density and the liquid sound velocity with the measured relative frequency shifts of $\mathrm{A}_{01}$ mode and $\mathrm{A}_{03}$ mode. These will be analyzed in following section.

\subsection{Viscosity Measurements with the $S_{0}$ Mode}

The responses of the $\mathrm{S}_{0}$ mode to the solutions of $\mathrm{NaBr}$ are similar to the ones of $\mathrm{NaCl}$ (Figure 3), the central frequency does not show apparent shifts due to their changing concentrations. However, the changes of the amplitude are related to the liquid's concentrations (Figure 4). It is because the frequency is mainly affected by the liquid density and sound velocity, which has no effects on the real part of the phase velocity of $\mathrm{S}_{0}$ mode (Equation (6)) [40]. The amplitude of the $\mathrm{S}_{0}$ mode in $\mathrm{NaBr}$ solution (Figure 4) shows that energy losses increase with the concentration, which is caused by the density and the viscosity of the liquid (Equation (7)). This can be used to decouple the density and the viscosity of the solution. 
Figure 4. The responses of the $\mathrm{S}_{0}$ mode to different concentrations of $\mathrm{NaBr}$ solutions.

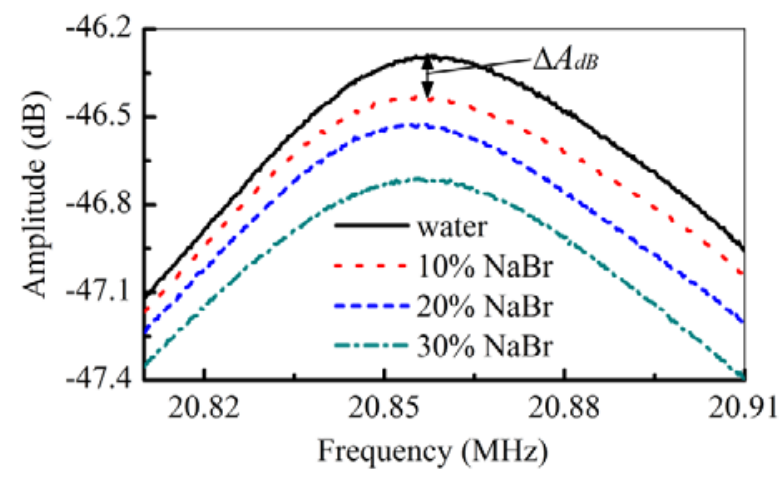

\section{Results and Discussions}

\subsection{To Decouple the Density and the Acoustic Sound Velocity with the $A_{01}$ Mode and $A_{03}$ Mode}

In order to specify the liquid with high selectivity, we attempted to decouple the liquid density and the liquid sound velocity with the measured frequency shifts of $\mathrm{A}_{01}$ mode and $\mathrm{A}_{03}$ mode. Based on the Equation (4), two steps are undertaken in order to decouple the density and the sound velocity of a liquid as follows:

(1) Determine the constant $B_{i}$ by measuring the frequency response of the reference liquid. Here, the water works as the reference liquid, the values of $B_{1}$ and $B_{3}$ are $14,767.3 \mathrm{~N} / \mathrm{m}$ and $101,642.8 \mathrm{~N} / \mathrm{m}$ respectively.

(2) Get the physical parameters $\left(\rho_{L}, c_{L}\right)$ for a unknown liquid by measuring $c_{P i}$. All other parameters $\left(B_{i}, \lambda_{i}, M\right)$ in Equation (1) have already been calculated or measured.

The first decoupled result using Equation (1) is the $\mathrm{NaCl}$ solution with different concentrations, as measured in Figure 3. Comparing the measured density and sound velocity with the values from the literature [32], the two results are close, as shown in Figure 5.

Figure 5. The decoupled density and sound velocity of $\mathrm{NaCl}$ solutions and other solutions using measurements in $\mathrm{A}_{01}$ mode and $\mathrm{A}_{03}$ mode.

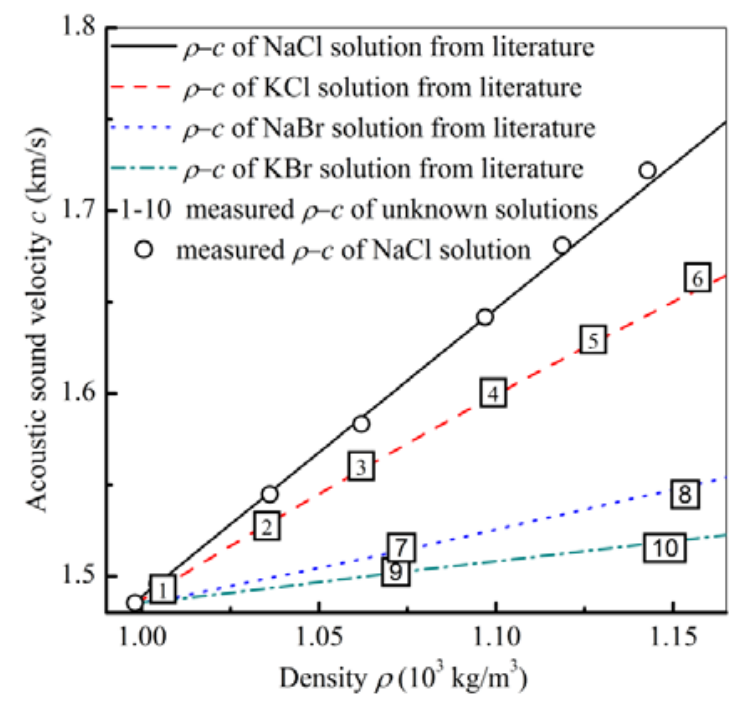


Based on the same process, the density and the sound velocity for other three different species solutions with different concentrations were also decoupled and are shown in Figure 5. Compared with the values from the literature [32], No. 1-No. 6 solutions are $\mathrm{KCl}$ solutions, No. 7 and No. 8 solutions are $\mathrm{NaBr}$ solutions, and No. 9 and No. 10 solutions are $\mathrm{KBr}$ solutions. Aside from decoupled density and sound velocity, the species of the solutions can be identified by comparing the measured values with the already known values [32]. After these density and sound velocity being decoupled using $\mathrm{A}_{01}$ mode and $\mathrm{A}_{03}$ mode, the liquid are determined with higher selectivity compared with only $\mathrm{A}_{01}$ mode measurement.

For Nos. 6, 8, and 10 solutions, the decoupled densities (Figure 5) are almost the same because the relative frequency shifts are very close (Table 2). For these solutions with adjacent density, sound velocities become the main factors affecting the frequency shifts in the $\mathrm{A}_{03}$ mode (Table 2). With the relations of the absolute frequency shifts: $\Delta f / f_{\mathrm{No} .6}>\Delta f / f_{\mathrm{No} .8}>\Delta f / f_{\mathrm{No} .10}$ (Table 2), the decoupled sound velocity of these three solutions have the following relation: $\mathrm{c}_{\mathrm{No} .6}>\mathrm{c}_{\mathrm{No} .8}>\mathrm{c}_{\text {No.10 }}$ (Figure 5).

\subsection{The Linear Response of (Density $\times$ Viscosity) $)^{0.5}$ Changing with the Item of Amplitude Shifts $\left(\Delta A_{d B}\right)$ in $S_{0}$ Mode}

The item (density $\times$ viscosity) $)^{0.5}$ changes almost linearly with the item of amplitude shifts $\left(\Delta A_{\mathrm{dB}}\right)$, and the linear fitting coefficient is about $1.02 \pm 0.09\left(\mathrm{~dB} /\left(\mathrm{kg} \cdot \mathrm{m}^{-2} \cdot \mathrm{s}^{-0.5}\right)\right)$, such as the $\mathrm{NaBr}$ and $\mathrm{NaCl}$ solutions (Figure 6). Beside the density and the viscosity, other parameters, such as the liquid's conductivity, affect the amplitude shifts. This is maybe the reason why the high linearity of $(\text { density } \times \text { viscosity })^{0.5}$ doesn't change with the amplitude shift. However, by analyzing the amplitude shifts $\left(\triangle A_{\mathrm{dB}}\right)$ of the $\mathrm{S}_{0}$ mode, the viscosity of the solution can be derived. With the determined density by measuring the frequency response of the $\mathrm{A}_{01}$ and $\mathrm{A}_{03}$ modes, the viscosity will be determined by checking the amplitude response in the $\mathrm{S}_{0}$ mode.

Figure 6. Amplitude shifts versus $\left(\right.$ density $\times$ viscosity ${ }^{0.5}$ for the $\mathrm{NaCl}$ and $\mathrm{NaBr}$ solutions in the $\mathrm{S}_{0}$ mode.

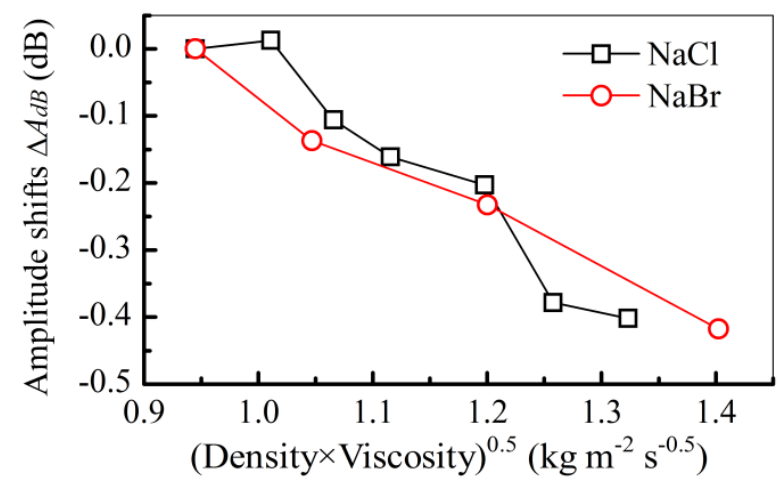

\section{Conclusions}

In this study, for the first time to our knowledge, the density, sound velocity, and viscosity of the liquid are obtained simultaneously based on the measurements of the same solution sample volume with a Lamb wave sensor. When the frequency shifts of the $\mathrm{A}_{01}$ and $\mathrm{A}_{03}$ modes are combined, the density and sound velocity are decoupled. This happens because the phase velocity of the $A_{01}$ mode is 
far from the sound velocity of the liquid and the phase velocity of the $\mathrm{A}_{03}$ mode is close to the sound velocity of the liquid. Viscosity is obtained by measuring the amplitude of the $\mathrm{S}_{0}$ mode, which is especially suitable for Newtonian fluids. Effects of the aqueous electrolytic solutions' conductivity on these three modes are not clearly observed. The term (density $\times$ viscosity) ${ }^{0.5}$ doesn't show high linearity as a function of amplitude shifts.

However, with this multi-parameter detection method, unknown solutions such as aqueous electrolytic solutions can be distinguished successfully and with high selectivity. Results clearly indicate that the multi-modes of a micro Lamb wave sensor are promising in the investigation of molecular thermodynamics, adiabatic compressibility, and molecular label free detection, among others.

\section{Acknowledgement}

The authors gratefully acknowledge the technical support from Engineers Jean-Yves Rauch of Femto-st, Pascal Blind of CTMN (Besançon), and Feng Li of CIOMP. Parts of the present work were financially supported by the National Natural Science Foundation of China (Nos. 11034007, 60971025) and the Chinese Academy of Sciences Innovation Program (KJCX2-YW-H18).

\section{References}

1. Fu, Y.Q.; Luo, J.K.; Du, X.Y.; Flewitt, A.J.; Li, Y.; Markx, G.H.; Walton, A.J.; Milne, W.I. Recent developments on $\mathrm{ZnO}$ films for acoustic wave based bio-sensing and microfluidic applications: A review. Sens. Actuators B 2010, 143, 606-619.

2. Hager, H.E. Fluid property evaluation by piezoelectric crystals operating in the thickness shear mode. Chem. Eng. Commun. 1986, 43, 25-38.

3. Kondoh, J.; Shiokawa, S. Measurements of conductivity and $\mathrm{pH}$ of liquid using surface acoustic wave devices. Jpn. J. Appl. Phys. 1992, 31, 82-84.

4. Lau, O.-W.; Shao, B.; Zhang, W. Evaluation of methods to minimize effects of liquid viscosity and density on the oscillating frequencies of thickness-shear-mode (TSM) piezoelectric resonators. Anal. Chim. Acta 1995, 312, 217-222.

5. Arapan, L.; Alexieva, G.; Avramov, I.D.; Radeva, E.; Strashilov, V.; Katardjiev, I.; Yantchev, V. Highly mass-sensitive thin Film Plate Acoustic Resonators (FPAR). Sensors 2011, 11, 6942-6953.

6. Sarvazyan, A.P. Ultrasonic velocimetry of biological compounds. Annu. Rev. Biophys. Biophys. Chem. 1991, 20, 321-342.

7. Martin, S.J.; Ricco, A.J.; Niemczyk, T.M.; Frye, G.C. Characterization of SH acoustic plate mode liquid sensors. Sens. Actuators 1989, 20, 253-268.

8. Montagut, Y.; García, J.V.; Jiménez, Y.; March, C.; Montoya, Á.; Arnau, A. Validation of a phase-mass characterization concept and interface for acoustic biosensors. Sensors 2011, 11, 4702-4720.

9. Kovacs, G.; Vellekoop, M.J.; Haueis, R.; Lubking, G.W.; Venema, A. A love wave sensor for (bio)chemical sensing in liquids. Sens. Actuators A 1994, 43, 38-43.

10. Newton, M.; Roach, P.; McHale, G. ST quartz acoustic wave sensors with sectional guiding layers. Sensors 2008, 8, 4384-4391. 
11. Länge, K.; Rapp, B.; Rapp, M. Surface acoustic wave biosensors: A review. Anal. Bioanal. Chem. 2008, 391, 1509-1519.

12. Rocha-Gaso, M.-I.; March-Iborra, C.; Montoya-Baides, A.; Arnau-Vives, A. Surface generated acoustic wave biosensors for the detection of pathogens: A review. Sensors 2009, 9, 5740-5769.

13. Voiculescu, I.; Nordin, A.N. Acoustic wave based MEMS devices for biosensing applications. Biosens. Bioelectron. 2012, 33, 1-9.

14. Priego Capote, F.; Luque de Castro, M. Ultrasound in analytical chemistry. Anal. Bioanal. Chem. 2007, 387, 249-257.

15. Cooper, M. Label-free screening of bio-molecular interactions. Anal. Bioanal. Chem. 2003, 377, 834-842.

16. Rocha-Gaso, M.-I.; March-Iborra, C.; Montoya-Baides, Á.; Arnau-Vives, A. Surface generated acoustic wave biosensors for the detection of pathogens: A review. Sensors 2009, 9, 5740-5769.

17. Rapp, B.; Gruhl, F.; Länge, K. Biosensors with label-free detection designed for diagnostic applications. Anal. Bioanal. Chem. 2010, 398, 2403-2412.

18. Hino, R.; Esashi, M.; Tanaka, S. Antisymmetric-mode Lamb wave methanol sensor with edge reflectors for fuel cell applications. In Proceedings of the 23rd IEEE International Conference on Micro Electro Mechanical Systems, Technical Digest (MEMS 2010), Hong Kong, 24-28 January 2010; pp. 871-874.

19. Latif, U.; Mujahid, A.; Afzal, A.; Sikorski, R.; Lieberzeit, P.; Dickert, F. Dual and tetraelectrode QCMs using imprinted polymers as receptors for ions and neutral analytes. Anal. Bioanal. Chem. 2011, 400, 2507-2515.

20. Raimbault, V.; Rebiere, D.; Dejous, C.; Guirardel, M.; Lachaud, J.L. Molecular weight influence study of aqueous poly(ethylene glycol) solutions with a microfluidic Love wave sensor. Sens. Actuators B 2010, 144, 318-322.

21. Herrmann, F.; Hahn, D.; Büttgenbach, S. Separate determination of liquid density and viscosity with sagittally corrugated Love-mode sensors. Sens. Actuators A 1999, 78, 99-107.

22. Doy, N.; McHale, G.; Newton, M.I.; Hardacre, C.; Ge, R.; MacInnes, J.M.; Kuvshinov, D.; Allen, R.W. Small volume laboratory on a chip measurements incorporating the quartz crystal microbalance to measure the viscosity-density product of room temperature ionic liquids. Biomicrofluidics 2010, doi:10.1063/1.3353379.

23. Mitsakakis, K.; Tsortos, A.; Kondoh, J.; Gizeli, E. Parametric study of SH-SAW device response to various types of surface perturbations. Sens. Actuators B 2009, 138, 408-416.

24. Oita, I.; Halewyck, H.; Thys, B.; Rombaut, B.; Vander Heyden, Y.; Mangelings, D. Microfluidics in macro-biomolecules analysis: Macro inside in a nano world. Anal. Bioanal. Chem. 2010, 398, 239-264.

25. Laurent, T.; Bastien, F.O.; Pommier, J.-C.; Cachard, A.; Remiens, D.; Cattan, E. Lamb wave and plate mode in $\mathrm{ZnO} /$ silicon and AlN/silicon membrane: Application to sensors able to operate in contact with liquid. Sens. Actuators A 2000, 87, 26-37.

26. Jia, H.; Duhamel, R.; Manceau, J.F.; de Labachelerie, M.; Bastien, F. Improvement of Lamb waves sensors: Temperature sensitivity compensation. Sens. Actuators A 2005, 121, 321-326. 
27. Choujaa, A.; Tirole, N.; Bonjour, C.; Martin, G.; Hauden, D.; Blind, P.; Cachard, A.; Pommier, C. AlN/silicon Lamb-wave microsensors for pressure and gravimetric measurements. Sens. Actuators A 1995, 46, 179-182.

28. Moll, J.; Golub, M.V.; Glushkov, E.; Glushkova, N.; Fritzen, C.-P. Non-axisymmetric Lamb wave excitation by piezoelectric wafer active sensors. Sens. Actuators A 2012, 174, 173-180.

29. Rizwaan, A. Time-space analysis of a surface-bonded piezoelectric film's response with Lamb wave for diagnostics. Sens. Actuators A 2010, 161, 12-22.

30. Li, F.; Wu, Y.; Manceau, J.-F.; Bastien, F. Temperature compensation of Lamb wave sensor by combined antisymmetric mode and symmetric mode. Appl. Phys. Lett. 2008, 92, 074101:1-074101:3.

31. Martin, B.A.; Wenzel, S.W.; White, R.M. Viscosity and density sensing with ultrasonic plate waves. Sens. Actuators A 1990, 22, 704-708.

32. Freyer, E.B. Sonic studies of the physical properties of liquids. II. The velocity of sound in solutions of certain alkali halides and their compressibilities. J. Am. Chem. Soc. 1931, 53, $1313-1320$.

33. White, R.M.; Voltmer, F.W. Direct piezoelectric coupling to surface elastic waves. Appl. Phys. Lett. 1965, 7, 314-316.

34. Zhou, L.; Manceau, J.-F.; Bastien, F. Influence of gases on Lamb waves propagations in resonator. Appl. Phys. Lett. 2009, 95, 223505-223507.

35. Zhou, L.; Manceau, J.-F.; Bastien, F. Interaction between gas flow and a Lamb waves based microsensor. Sens. Actuators A 2012, 181, 1-5.

36. Chen, Z.; Han, T.; Shi, W.; Ji, X.; Li, L. Separate determination of liquid properties with Lamb wave devices. In Proceedings of the 2006 IEEE International Frequency Control Symposium and Exposition, Miami, FL, USA, 4-7 June 2006; Volumes 1-2, pp. 322-327.

37. Moroney, R.M.; White, R.M.; Howe, R.T. Microtransport induced by ultrasonic Lamb waves. Appl. Phys. Lett. 1991, 59, 774-776.

38. Nguyen, N.T.; White, R.M. Design and optimization of an ultrasonic flexural plate wave micropump using numerical simulation. Sens. Actuators A 1999, 77, 229-236.

39. Sayar, E.; Farouk, B. Acoustically generated flows in microchannel flexural plate wave sensors: Effects of compressibility. Sens. Actuators A 2011, 171, 317-323.

40. Osborne, M.F.M.; Hart, S.D. Transmission, reflection, and guiding of an exponential pulse by a steel plate in water. I. theory. J. Acoust. Soc. Am. 1945, 17, 1-18.

41. Leckey, C.A.C.; Rogge, M.D.; Miller, C.A.; Hinders, M.K. Multiple-mode Lamb wave scattering simulations using 3D elastodynamic finite integration technique. Ultrasonics 2012, 52, 193-207.

42. Zhu, Z.; Wu, J.; Li, J.; Zhou, W. A general dispersion relation for Lamb-wave sensors with liquid-layer loading. Sens. Actuators A 1995, 49, 79-84.

(C) 2012 by the authors; licensee MDPI, Basel, Switzerland. This article is an open access article distributed under the terms and conditions of the Creative Commons Attribution license (http://creativecommons.org/licenses/by/3.0/). 